\title{
Recent introductions
}

\section{Wipe that DNA right out of your plastic and glassware containers.}

\section{Multiskan Spectrum}

Thermo Labsystems

www.thermo.com

A new UV/VIS microplate and cuvette spectrophotometer

The Multiskan Spectrum combines both standard UV/visible spectrophotometer cuvette reading capabilities and advanced microtitre plate reading into a convenient, compact instrument. The dual-beam cuvette reader offers both sample and reference beams, for enhanced, long-term stability. Multiskan Spectrum can make quick absorbance measurements in a cuvette between the cycles of a microtitre plate enzyme kinetics experiment. The cuvette port's short measurement cycle optimizes enzyme kinetics conditions before transferring kinetic protocols to microtitre plate format.

\section{Montage DNA Gel Extraction Kit}

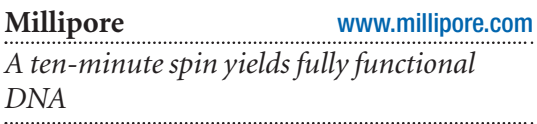

The latest addition to the Montage range of genomics sample prep kits is Montage DNA Gel Extraction Kit for the recovery of fully functional DNA from agarose gel slices in a single 10-minute spin. DNA fragments that are $100-10,000$ base pairs in size may be efficiently recovered ready for sequencing, cloning or labelling with no additional purification. The Montage DNA Gel Extraction kit is available as a 50-pack containing 50 Montage DNA Gel Extraction (formerly Ultrafree-DA) centrifugal devices and, for added convenience, a bottle of $500 \mathrm{ml}$ of $50 \times$ modified TAE buffer for gel casting and running. Each centrifugal device is ready to use, consisting of three pre-assembled components: a gel nebulizer, a sample filter cup and a collection microcentrifuge tube. The specially formulated modified TAE buffer contains one-tenth the standard EDTA concentration to minimize interference with downstream applications.

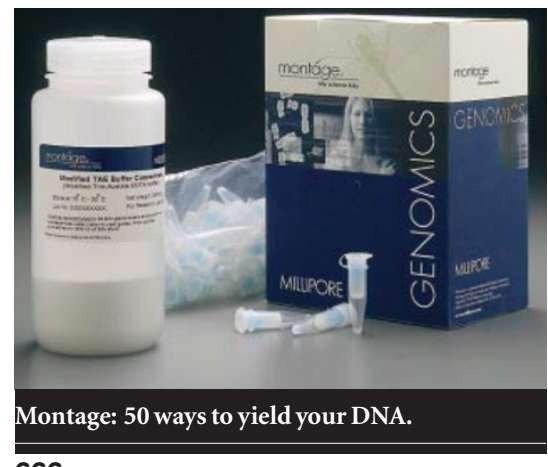

MiniBeadbeater-96

Biospec Products

High-capacity cell disrupter

Designed for high-throughput analytical screening, the MiniBeadbeater-96 will disrupt up to 128 samples of spores, microorganisms, plant and animal tissue or soil samples at a time. Using the same homogenization process as its predecessors, MiniBeadbeater-1 and -8, one or two deep-well microtitre plates containing sample and a large number of tiny ceramic beads are violently shaken for 2-3 minutes to achieve over $90 \%$ cell disruption. The plates and beads are disposable, making them suitable for PAGE, PCR and Probe applications where cross-contamination between samples cannot be tolerated. Compatible with phenol, Gu-SCN and commercial kit solutions, RNA/DNA is quickly recovered at high yield by doing cell disruption and nucleic acid extraction simultaneously.

\section{TRobot}

Biometra www.biometra.com Let the machines take the strain

The Biometra TRobot is designed specifically for integration in robotic systems. The thermocycler module is separated from the controller unit for a small footprint. The motorized heated lid ensures automated workflow and reliable sealing of plates. TRobot features a motorized plate lifter. Oncelifted the plate can be easily removed by a robotic arm. All features of the TRobot are controlled over its serial port. Integration in existing software environments (of individual robotic stations) is very easy. In addition to controlling standard features, the TRobot can provide extensive status messages. A program for operating up to 16 TRobots from a computer is also available. The TRobot comes with 96- or 384- well block.

\section{BD TALON HT}

BD Biosciences Clontech www.clontech.com In His name

The BD TALON HT 96-Well Plate is intended for studies needing rapid or high-throughput purification of histidine-tagged proteins. Each well plate is pre-loaded with $200 \mathrm{mg}$ of BD TALON Superflow Resin in each well of a 96-well filtration plate. That provides enough resin to bind up to $1 \mathrm{mg}$ of histidine-tagged protein per well. The plate is provided with convenient top and bottom seals for shipping and storage, as well as a collection plate that can be used for receiving the eluted His-tagged proteins and for storage of samples.

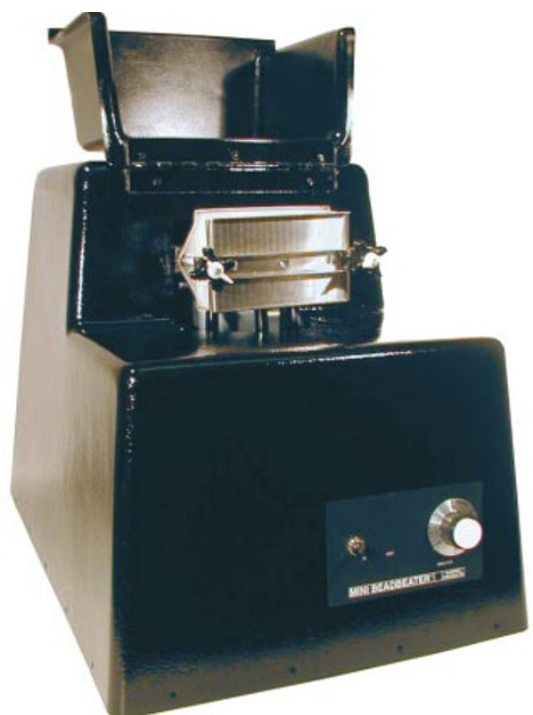

A bit shaky: MiniBeadbeater-96.

NucleasEliminator wipes

AMRESCO

www.amresco-inc.com

Nucleases removed at a stroke

NucleasEliminator Wipes are a handy and convenient way to remove RNase and DNase contamination. The wipes are ready to use, single-use moist towelettes packed singly in a foil pouch. Active ingredient is AMRESCO's NucleasEliminator solution. Suitable for removing nuclease from any plastic and glassware surfaces without contaminating the experiment, these wipes are recommended for researchers performing PCR and delicate electrophoresis reactions and experiments. Each box contains 25 wipes and can be stored at room temperature.

These notes are compiled in the Nature office from information provided by the manufacturers. 